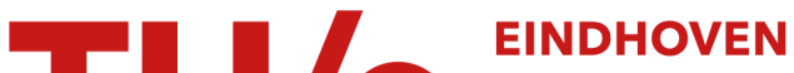 UNIVERSITY OF TECHNOLOGY
}

\section{Selection of actuators and sensors for active surge control}

\section{Citation for published version (APA):}

Wal, van de, M. M. J., Willems, F. P. T., \& Jager, de, A. G. (1997). Selection of actuators and sensors for active surge control. In Proceedings of the 1997 IEEE international conference on control applications : October 5-7, 1997, Hartford, Connecticut, USA (pp. 121-126). Institute of Electrical and Electronics Engineers. https://doi.org/10.1109/CCA.1997.627493

DOI:

10.1109/CCA.1997.627493

Document status and date:

Published: 01/01/1997

\section{Document Version:}

Publisher's PDF, also known as Version of Record (includes final page, issue and volume numbers)

\section{Please check the document version of this publication:}

-A submitted manuscript is the version of the article upon submission and before peer-review. There can be important differences between the submitted version and the official published version of record. People interested in the research are advised to contact the author for the final version of the publication, or visit the $\mathrm{DOI}$ to the publisher's website.

- The final author version and the galley proof are versions of the publication after peer review.

- The final published version features the final layout of the paper including the volume, issue and page numbers.

Link to publication

\section{General rights}

Copyright and moral rights for the publications made accessible in the public portal are retained by the authors and/or other copyright owners and it is a condition of accessing publications that users recognise and abide by the legal requirements associated with these rights.

- Users may download and print one copy of any publication from the public portal for the purpose of private study or research.

- You may not further distribute the material or use it for any profit-making activity or commercial gain

- You may freely distribute the URL identifying the publication in the public portal.

If the publication is distributed under the terms of Article $25 f a$ of the Dutch Copyright Act, indicated by the "Taverne" license above, please follow below link for the End User Agreement:

www.tue.nl/taverne

Take down policy

If you believe that this document breaches copyright please contact us at:

openaccess@tue.nl

providing details and we will investigate your claim. 


\title{
Selection of Actuators and Sensors for Active Surge Control
}

\author{
Marc van de Wal, Frank Willems, and Bram de Jager \\ Faculty of Mechanical Engineering, Eindhoven University of Technology \\ P.O. Box 513, 5600 MB Eindhoven, The Netherlands \\ M.M.J.v.d.Wal@wfw.wtb.tue.nl
}

\begin{abstract}
Active control is one way to cope with surge in compression systems. For this, a suitable combination of actuators and sensors must be selected. In this paper, the goal is to find a combination fulfilling the condition that a controller exists, which stabilizes the nominal model in the face of disturbances and actuator limitations. For a collection of linearizations of the nonlinear system, this condition is quantified as an $\mathcal{H}_{\infty}$ norm bound on the closed-loops. Among the proposed actuators and sensors, the close-coupled valve and mass flow sensor are the most promising for the considered system, but a movable wall should be added to meet the specifications.
\end{abstract}

Keywords: actuator and sensor selection; compressor surge control; $\mathcal{H}_{\infty}$ control; nonlinear systems.

\section{Introduction}

The usability of compressors is hampered by rotating stall and surge. These aerodynamic flow instabilities may even lead to hazardous conditions due to large mechanical and thermal loads. Hence, it is desirable to enlarge the region of stable compressor operation. Among the various ways to achieve this (see [2] for a survey), active control is a recent and promising option. This paper will focus on suppression of surge in the face of exogenous disturbances and actuator limitations. Surge is characterized by large amplitude fluctuations of pressure rise and mass flow. This one-dimensional phenomenon tends to occur in the operating region with relatively small mass flows and results in a limit cycle oscillation.

Besides modeling and controller design, a major issue in control system design is the selection of an appropriate number, place, and type of actuators (inputs) and sensors (outputs). This is called Input Output (IO) selection. The set of actuators and sensors (IO set) affects aspects like achievable performance, system complexity, hardware costs, and maintenance effort. Due to the possibly huge number of candidate IO sets, favorable ones may be overlooked if IO selection only relies on physical insight and experience. So, a systematic method will be helpful; see [5] for a brief survey of IO selection methods.

The method recently proposed in [6] is employed, since it puts less restrictions on the control problem formulation than many other methods. Like the majority of IO selection methods, it is restricted to linear systems. Therefore, the nonlinear compression system model is linearized in various operating points. IO selection should at least aim at finding the IO set(s) for which a controller exists that stabilizes the nominal model. For the considered case, additional performance specifications involve limiting the deviations of control inputs, mass flow, and pressure from their nominal values in the face of disturbances. Therefore, the IO selection method aims at finding the
IO set(s) for which a stabilizing controller exists that achieves a desired Nominal Performance (NP) level. A requirement on the closed-loop's $\mathcal{H}_{\infty}$ norm quantifies this goal. Three candidate actuators and four sensors are proposed, which are also among those examined in [4]. This yields 105 candidate IO sets, among which the full IO set including all actuators and sensors and 12 IO sets with a single actuator and sensor (" $1 \times 1$ IO set"). For various reasons (mainly costs), the accepted IO set with the least actuators and sensors is preferable for control.

The two main contributions of this paper are the following. First, the practical usefulness of the IO selection method from [6] is further evaluated. It is investigated if the method can cope with nonlinear systems and performance specifications which are not directly linked to the $\mathcal{H}_{\infty}$ norm setting. Second, the IO selection for the compressor control problem is believed to be more rigorous than previously found in literature. For instance, [4] considers proportional output feedback with $1 \times 1$ IO sets on the one hand and state feedback with single actuators under disturbances on the other. The IO selection method in the present paper allows for dynamic output feedback and incorporates performance specifications and disturbance characterizations in a standard framework.

The paper is organized as follows. Section 2 sketches the control problem and proposes the candidate actuators and sensors. Section 3 summarizes the key ideas of the IO selection method and Section 4 quantifies the control goal. $\mathcal{H}_{\infty}$ optimizations in Section 5 create insight on the importance of each actuator and sensor. Section 6 provides the IO selection results, while Section 7 shows simulations for two promising IO sets. Finally, Section 8 draws conclusions and recommends topics for further research. Readers who are interested in numerical values of model and design parameters and details on the IO selection method and the application are encouraged to request the extended report [7].

\section{The Compressor Control Problem}

The investigated compression system is schematized in Fig. 1. In the employed Greitzer lumped parameter model [3], the (radial) compressor and its ducting are modeled as an actuator disk accounting for the pressure rise and a constant area pipe accounting for the fluid dynamics. The compressed fluid is discharged into a plenum and delivered to a process, represented by a throttle across which the pressure drops.

In general, the state of a compression system is determined by four nondimensional variables: the compressor mass flow, the plenum pressure rise, the rotor speed, and the temperature (or a related variable, like efficiency). The last variable is usually not needed to characterize the surge region. The dimensional compressor mass flow $\dot{m}_{c}[\mathrm{~kg} / \mathrm{s}]$ and plenum pressure 


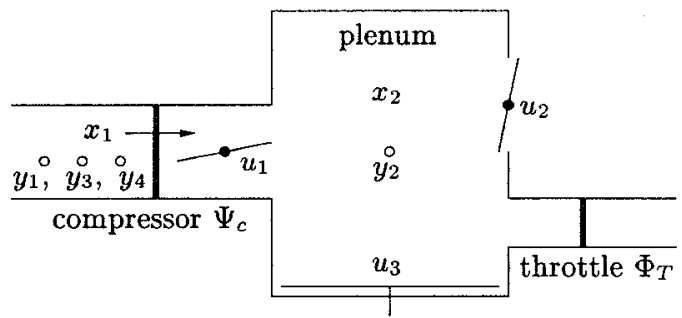

Figure 1: The compression system with candidate sensors and actuators

rise $\Delta p[\mathrm{~Pa}]$ are made nondimensional as follows:

$$
x_{1}=\frac{\dot{m}_{c}}{\rho_{a} A_{c} U_{T}} \quad \text { and } \quad x_{2}=\frac{\Delta p}{\frac{1}{2} \rho_{a} U_{T}^{2}}
$$

with $\rho_{a}\left[\mathrm{~kg} / \mathrm{m}^{3}\right]$ the ambient air density, $A_{c}\left[\mathrm{~m}^{2}\right]$ the compressor flow through area, and $U_{T}[\mathrm{~m} / \mathrm{s}]$ the rotor tip speed. In steady-state, $x_{1}, x_{2}$, and the rotor speed $n$ are connected via the speed line $\Psi_{c}$ (also called compressor characteristic) and only two of $x_{1}, x_{2}$, and $n$ are independent.

The original Greitzer model is restricted to constant speeds and Fig. 2 depicts $\Psi_{c}$ for five distinct values of $n$ [rev/min]. For a fixed speed, $\Psi_{c}$ is approximated by the following third order polynomial in $x_{1}$ :

$$
\Psi_{c}\left(x_{1}\right)=\Psi_{c_{0}}+H\left[1+\frac{3}{2}\left(\frac{x_{1}}{F}-1\right)-\frac{1}{2}\left(\frac{x_{1}}{F}-1\right)^{3}\right]
$$

The positive parameters $\Psi_{c_{0}}, H$, and $F$ are determined from data available for a laboratory set-up, see [7]. The investigated flow region for each speed is given by $x_{1_{0}} \in[F, 3 F]$, with $x_{1_{0}}$ the nominal mass flow and $x_{1_{0}}=2 F$ the flow where $\Psi_{c}$ peaks. Throughout this study, emphasis is put on $\Psi_{c}$ for the "central" speed $n_{3}=80,000[\mathrm{rev} / \mathrm{min}]$. For $x_{1_{0}} \in[F, 3 F]$, the equilibria $\left(x_{1_{0}}, x_{2_{0}}\right)$ on $\Psi_{c}$ are stable for [7]

$$
x_{1_{0}}>2 F-\frac{F^{3}}{3 H B^{2}}, \quad \text { with } \quad B=\frac{U_{T}}{2 a} \sqrt{\frac{V_{p}}{A_{c} L_{c}}}
$$

and $a[\mathrm{~m} / \mathrm{s}]$ the speed of sound, $V_{p}\left[\mathrm{~m}^{3}\right]$ the plenum volume, and $L_{c}[\mathrm{~m}]$ the compressor duct length. $B$ is often called the compressor stability parameter. For increasing $B$, the stable operating region decreases. The considered system has large $B$, ranging from 10 to 25 , and the second term on the right hand side of inequality (3) is approximately zero. So, the equilibria on most of the positively sloped part of $\Psi_{c}$ are unstable.

For $x_{2} \geq 0$, the throttle mass flow is modeled by:

$$
\Phi_{T}\left(x_{2}\right)=K_{T} \sqrt{x_{2}} .
$$

$K_{T}$ involves the throttle area, which is determined by downstream demands, e.g., constant pressure and/or mass flow delivery. In this study, $K_{T}$ is set so the intersection of $\Psi_{c}$ and $\Phi_{T}$ yields the desired operating point $\left(x_{1_{0}}, x_{2_{0}}\right)$.

The control goal is to guarantee stable compressor operation under disturbances and actuator limitations, locally around stationary nominal operating points $\left(x_{1_{0}}, x_{2_{0}}\right)$. This issue is particularly important for unstable nominal operating points. The variables which should remain small are collected in the column of regulated variables $z$ (see the system equations (5)). To make it possible to impose that the state trajectory should remain close to the nominal operating point, $z_{1}$ and $z_{2}$ represent the deviations of $x_{1}$ and $x_{2}$ from their nominal values. The control actions $u$ are limited and also included in $z$. The

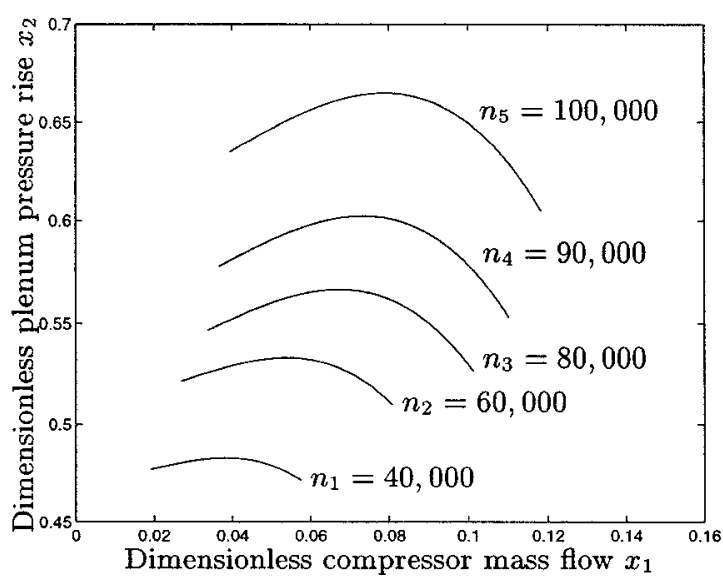

Figure 2: Compressor map with speed lines $\Psi_{\mathrm{c}}$ for various rotor speeds $n[\mathrm{rev} / \mathrm{min}]$ and $x_{1_{0}} \in[F, 3 F]$

following nonpersistent disturbances are considered, which are part of the exogenous variables $w$ : inlet pressure disturbances $w_{1}$, caused by upstream processes; outlet flow disturbance $w_{2}$, caused by downstream processes; compressor pressure disturbance $w_{3}$, e.g., rapidly fluctuating local phenomena due to turbulence in the rotor. Finally, $w$ includes sensor noise.

To monitor and manipulate system behavior, the control system should include actuators and sensors. Ideally, a single actuator and sensor suffice for local stabilization [7]. In practice, disturbances and actuator limitations play a role and stabilization with a $1 \times 1$ IO set may become impossible. This may be resolved by adding actuators and sensors, see Fig. 1. Four measured variables $y$ (sensors) are proposed: compressor mass flow $y_{1}$; plenum pressure rise $y_{2}$; total compressor face pressure $y_{3}$; static compressor face pressure $y_{4}$. As manipulated variables $u$ (actuators), the following three options are studied: close-coupled valve parameter $u_{1}$; bleed valve parameter $u_{2} ;$ movable wall velocity $u_{3}$. For $x_{1} \geq 0$ and $x_{2} \geq 0$, the nonlinear compression system model including all relevant variables then becomes:

$$
\begin{aligned}
& \dot{x}_{1}=B\left[\Psi_{c}\left(x_{1}\right)-x_{2}+w_{1}+w_{3}-\left(\frac{x_{1}}{c_{1}}\right)^{2} u_{1}\right] \\
& \dot{x}_{2}=\frac{1}{B}\left[x_{1}-\Phi_{T}\left(x_{2}\right)+w_{2}-c_{2} \sqrt{x_{2}} u_{2}\right]-\left(\alpha x_{2}+\beta\right) u_{3} \\
& z_{i}=x_{i}-x_{i_{0}}, \quad i=1,2 \\
& z_{i}=u_{i}-u_{i_{0}}, \quad i=3,4,5 \\
& y_{1}=x_{1}+w_{4} \\
& y_{2}=x_{2}+w_{5} \\
& y_{3}=x_{2}-\Psi_{c}\left(x_{1}\right)-\left(w_{1}+w_{3}\right)+\left(\frac{x_{1}}{c_{1}}\right)^{2} u_{1}+w_{6} \\
& y_{4}=-x_{1}^{2}+x_{2}-\Psi_{c}\left(x_{1}\right)-\left(w_{1}+w_{3}\right)+\left(\frac{x_{1}}{c_{1}}\right)^{2} u_{1}+w_{7} .
\end{aligned}
$$

The differential equations in (5) without $w$ and $u$ form the Greitzer model [3], which is based on conservation of mass in the plenum and conservation of momentum in the compressor duct. For more details on (5), see [7]. .

In the nonlinear model (5), $u_{1}$ and $u_{2}$ can only take positive values, since the valves can only cause pressure drops in the direction of the flow. Input $u_{2}$ could be interpreted as the available flow through area. This does not hold for $u_{1}$, owing to an input transformation [7] to create the input affine model (5). The nominal values $u_{1_{0}}$ and $u_{2_{0}}$ are chosen so the pressure drops across the valves can be larger or smaller than nominal and so the efficiency loss is acceptable: $u_{1_{0}}$ is set so the pressure drop across the close-coupled valve is $1 \%$ of the maximum pressure rise $\Psi_{c}(2 F)$ for a fully open valve; $u_{2_{0}}$ is set so the mass flow through the bleed valve is $1 \%$ of $2 F$. The presence of the close-coupled valve effectively modifies the shape of $\Psi_{c}$ 
resulting in an enlarged stable operating region of the openloop system: for speed $n_{3}$, the nominal operating points with $x_{1_{0}}>1.77 F$ instead of (3) are stable. For obvious reasons, the movable wall's nominal velocity $u_{3_{0}}$ is set to zero.

The remainder of the paper focuses on linearizations of (5) and from now on $x, w, u, z$, and $y$ apply to the corresponding variables for the linearization.

\section{The IO Selection Method}

To use the IO selection method from [6], the nonlinear plant (5) is linearized around various nominal operating points. The linearized plant $P$ is incorporated in the standard control system set-up of Fig. 3. The generalized plant $G$ is formed by extending $P$ with the design filters $V$ and $W$, see below. The linear controller is denoted $K$.

To characterize performance, system norms could be used to quantify how large the regulated variables $z$ (ideally zero) can get for a class of exogenous variables $w$. Here, the $\mathcal{H}_{\infty}$ norm is the focus, which for instance arises when minimizing the energy in $z$ for bounded-energy $w$. For a stable linear system $T$, the $\mathcal{H}_{\infty}$ norm is defined as:

$$
\|T\|_{\infty}:=\sup _{\omega} \bar{\sigma}(T(j \omega))
$$

with $\bar{\sigma}$ the maximum singular value of a matrix. A meaningful control problem formulation accounts for the relative magnitudes and importance of the components in $w$ and $z$ respectively. For this purpose, the diagonal transfer function matrices $V$ and $W$ are introduced. $V$ is called the shaping filter, since it is used here to characterize the magnitude and frequency dependence of $w$; in Fig. $3, \tilde{w}$ is a scaled version of the physical $w . W$ is called the weighting filter, as it can be used to indicate which components and frequencies in $z$ are to be suppressed; $\tilde{z}$ in Fig. 3 is a weighted version of $z$. Given $V$ and $W$, a desired NP level is expressed as an $\mathcal{H}_{\infty}$ norm bound: $\|M\|_{\infty}<\gamma$, with the transfer function matrix $M$ relating $\tilde{w}$ and $\tilde{z}$ and $\gamma$ a designer-specified positive scalar. For more details on control problem formulations in the set-up of Fig. 3 , see $[1,9]$.

For each IO set, the existence of a stabilizing controller $K$ achieving $\|M\|_{\infty}<\gamma$ can be checked by six conditions, which form the basis for the IO selection method. Only an interpretation of these conditions is given here; for details, see [9, Chapters $16 \& 17]$. To apply the conditions, the state-space description of $G$ must satisfy the standard $\mathcal{H}_{\infty}$ design assumptions [9, Chapter 17]. This is guaranteed for the compressor application and usually not restrictive for practical problems. Essentially, the six conditions involve the following checks:

1. Check if the open-loop direct feedthrough from $\tilde{w}$ to $\tilde{z}$ is not "too large," since a certain component of it is unaffected by $K$.

2,3. Check if a controller with full information on $w$ and the states $x$ (ideal output set) can meet $\|M\|_{\infty}<\gamma$.

4,5. Check if a controller with full access to $z$ and $x$ (ideal input set) can meet $\|M\|_{\infty}<\gamma$.

6. Based on results from $2-5$, check if the combination of input set and output set can yield $\|M\|_{\infty}<\gamma$.

The IO selection method proceeds as follows. First, $V$ and $W$ are chosen so the mathematical problem of constructing a stabilizing controller achieving $\|M\|_{\infty}<\gamma$ suitably represents

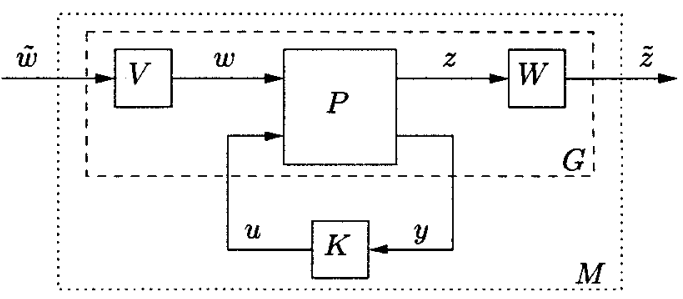

Figure 3: Standard control system set-up

the design specifications. Second, the candidate IO sets are subjected to the six conditions. As soon as one condition fails, the others need not be checked. In this way, a large number of IO sets can be assessed quickly, circumventing the timeconsuming process of (optimal) controller design and closedloop evaluation for each IO set. Functions from the MATLAB toolbox [1] form the basis for checking the conditions.

For nonlinear systems, an equivalent for the linear $\mathcal{H}_{\infty}$ control problem can be formulated. This is commonly referred to as nonlinear $\mathcal{H}_{\infty}$ control. As for linear systems, conditions for the existence of a stabilizing controller achieving a desired NP level $\gamma$ have been derived. In [8, Chapter 7], it is shown that the nonlinear $\mathcal{H}_{\infty}$ problem is locally solvable in a region around an equilibrium if (sufficient) the $\mathcal{H}_{\infty}$ problem for the linearization is solvable. Unfortunately, the size of this region cannot be specified, nor is it known a priori. For the compressor control problem, a range of equilibria (nominal operating points) rather than one equilibrium is of interest. Therefore, the IO selection conditions will be applied to a collection of linearizations for interesting operating points. Note, that IO sets accepted on this basis may not be suitable for control in other points than those investigated. For definite conclusions on the usability of the IO set for the nonlinear system, additional studies should be performed, e.g., closed-loop simulations.

\section{$4 \mathcal{H}_{\infty}$ Performance Specifications}

This section discusses the choice of the design filters $V$ and $W$, see also Table 1. The Helmholtz frequency $\omega_{H}=a \sqrt{A_{c} /\left(V_{p} L_{c}\right)}$ $[\mathrm{rad} / \mathrm{s}$ ] (the natural frequency of the oscillations of the gas in duct and plenum, in the absence of compressor and throttle) is used for scaling time and frequency-related parameters: $\tilde{t}=$ $t \omega_{H}, \tilde{\omega}=\omega / \omega_{H}$, with $\tilde{t}$ and $\tilde{\omega}$ nondimensional.

The shaping filters $V_{i}$ corresponding to the disturbances $w_{1}, w_{2}$, and $w_{3}$ are set according to:

$$
V_{i}=\frac{v_{i}}{s / \tilde{\omega}_{i}+1} \quad i=1,2,3
$$

which expresses the low-frequency character of the disturbances. Here, the choice of $\tilde{\omega}_{i}$ and $v_{i}$ is based on experience and intuition. If more physical knowledge or experimental data were available, a better choice could be made. Parameter $v_{1}$ is assumed $1 \%$ of the ambient pressure $p_{a}$ in nondimensional

Table 1: Performance specifications

\begin{tabular}{rccccccc}
\hline \multicolumn{3}{c}{ Shaping Filters $V$} & \multicolumn{3}{c}{ Weighting Filters $W$} \\
\hline$v_{1}:$ & $\frac{0.01 p_{a}}{\frac{1}{2} \rho_{a} U_{T}^{2}}$ & $\tilde{\omega}_{1}:$ & $\frac{20 \pi}{\omega_{H}}$ & $W_{1}:$ & $\frac{0.1}{x_{1}}$ & $\omega_{0}:$ & $10^{6}$ \\
$v_{2}:$ & $0.10 x_{1_{0}}$ & $\tilde{\omega}_{2}:$ & $\frac{20 \pi}{\omega_{H}}$ & $W_{2}:$ & $\frac{0.1}{x_{2}}$ & $\tilde{\omega}_{4}: \frac{200 \pi}{\omega_{H}}$ \\
$v_{3}:$ & $0.05 x_{2_{0}}$ & $\tilde{\omega}_{3}:$ & $\frac{2 \pi n N_{b}}{60 \omega_{H}}$ & $\rho_{1}:$ & $4.510^{-2}$ & $\tilde{\omega}_{5}: \frac{200 \pi}{\omega_{H}}$ \\
$V_{4}:$ & $\frac{1.1010^{-3}}{\rho_{a} A_{c} U_{T}}$ & & & $\rho_{2}: 2.610^{-3}$ & $\tilde{\omega}_{6}: \frac{100 \pi}{\omega_{H}}$ \\
$V_{5,6,7}:$ & $\frac{220}{\frac{1}{2} \rho_{a} U_{T}^{2}}$ & & & $\rho_{3}: 7.010^{-2}$ & & \\
\hline
\end{tabular}


form, while $v_{2}$ and $v_{3}$ are fixed fractions of the nominal mass flow and pressure rise respectively. For the compressor pressure disturbance, $\tilde{\omega}_{3}$ is related with the rotor speed $n$ and the number of rotor blades $N_{b}$. The exogenous variables also include sensor noises $w_{4}, \ldots, w_{7}$. Under the assumption that the magnitudes are the same for all frequencies, $V_{4}, \ldots, V_{7}$ are constants related to RMS values from experimental data.

As an initial attempt, the weights $W_{1}$ and $W_{2}$ for $z_{1}$ and $z_{2}$ are taken constant. By scaling, the denominators in $W_{1}$ and $W_{2}$ account for the relative magnitudes of the components in $z$ (equivalently for $W_{3}, W_{4}$, and $W_{5}$ ). From simulations with the nonlinear plant and linear controllers $K$ for exponentially decaying disturbances (see Section 7), it was observed that setting $W_{1}=W_{2}=0$ may yield responses that end up in a different closed-loop equilibrium than the nominal operating point $\left(x_{1_{0}}, x_{2_{0}}\right)$. If $z_{1}$ and $z_{2}$ are weighted, the system trajectory returns to $\left(x_{1_{0}}, x_{2_{0}}\right)$ and remains closer to this point, for which $K$ was computed.

The regulated variables include actuator weights $z_{3}, z_{4}$, and $z_{5}$ with corresponding high-pass weighting filters:

$$
W_{i+2}=\frac{\rho_{i}}{\bar{u}_{i}} \frac{s / \tilde{\omega}_{i+3}+1}{s / \omega_{0}+1}, \quad i=1,2,3
$$

The allowable operating ranges of the inputs $u$ in the linearization are given by $u_{i} \in\left[-\bar{u}_{i}, \bar{u}_{i}\right], i=1,2,3$, with $\bar{u}_{1}=u_{1_{0}}-1$ and $\bar{u}_{2}=u_{2_{0}}$. The maximally allowable nondimensional movable wall velocity is assumed $\bar{u}_{3}=1.0 / \frac{V_{p} \omega_{H}}{A_{p}}$, with $A_{p}$ the movable wall area. The displacement of the movable wall is limited by the construction. This could be accounted for, e.g., by a modified $W_{5}$ (see [7]), but (8) is suitable for the case considered here. The parameters $\tilde{\omega}_{4}, \tilde{\omega}_{5}$, and $\tilde{\omega}_{6}$ represent the actuator bandwidths. Assuming that $u_{3}$ is constructed as a relatively slowly moving plate, $\tilde{\omega}_{6}$ is relatively small. In case of a loudspeaker, $\tilde{\omega}_{6}$ may be larger. A natural choice is $\omega_{0}=\infty$, but $\omega_{0}=10^{6}$ allows a state-space representation of $G$. Up till $\omega=10^{5}$, this results in essentially the same characteristics of the weighting filters as for $\omega_{0}=\infty$.

Parameters $\rho_{3}, \rho_{4}$, and $\rho_{5}$ should be chosen so $u$ stays within its limits for the considered disturbances. This is not straightforward, since bounding the amplitudes of $z$ is not directly linked to the $\mathcal{H}_{\infty}$ norm setting. As an approximation, $\rho_{3}, \rho_{4}$, and $\rho_{5}$ are determined by iterative $\mathcal{H}_{\infty}$ controller optimizations and closed-loop simulations. This is done for the full IO set, for speed $n_{3}$, and for disturbances (9). The nominal operating point with $x_{1_{0}}=F$ is studied, for which control is expected to be the most difficult: for the unstable region and decreasing $x_{1_{0}}$, the pole with the largest real part shifts further to the right in the complex plane. The resulting $\rho$-parameters also work well for other $x_{1_{0}} \in[F, 3 F]$ and corresponding controllers.

\section{$5 \quad \mathcal{H}_{\infty}$ Optimizations}

By performing $\mathcal{H}_{\infty}$ optimizations for the eight IO sets in Table 2, insight is gained on the importance of each candidate actuator and sensor. The focus is on speed $n_{3}$. For each IO set, the design filters from Section 4 are used to construct $\mathcal{H}_{\infty}$ optimal controllers for 21 evenly spaced points in the range $x_{1_{0}} \in[F, 3 F]$. The optimal closed-loop norms $\|M\|_{\infty}$ are depicted in Fig. 4 and Fig. 5. Recall, that large norms imply a large gain between exogenous and regulated variables, i.e., bad performance. Since eliminating actuators and sensors will never improve the best achievable performance, the optimal $\mathcal{H}_{\infty}$ norm for the full IO set will never be larger than
Table 2: Typical IO sets subjected to $\mathcal{H}_{\infty}$ optimizations

\begin{tabular}{cccl}
\hline IO set & Outputs & Inputs & Characteristic \\
\hline \hline 1 & $y_{1} y_{2} y_{3} y_{4}$ & $u_{1} u_{2} u_{3}$ & full IO set \\
2 & $y_{1} y_{2} y_{3} y_{4}$ & $u_{1}$ & close-coupled valve \\
3 & $y_{1} y_{2} y_{3} y_{4}$ & $u_{2}$ & bleed valve \\
4 & $y_{1} y_{2} y_{3} y_{4}$ & $u_{3}$ & movable wall \\
5 & $y_{1}$ & $u_{1} u_{2} u_{3}$ & compressor mass flow \\
6 & $y_{2}$ & $u_{1} u_{2} u_{3}$ & plenum pressure rise \\
7 & $y_{3}$ & $u_{1} u_{2} u_{3}$ total compressor face pressure \\
8 & $y_{4}$ & $u_{1} u_{2} u_{3}$ & static compressor face pressure \\
\hline
\end{tabular}

the $\mathcal{H}_{\infty}$ norm for IO sets with fewer actuators and sensors ("smaller" IO sets).

Figure 4 compares $\|M\|_{\infty}$ for IO sets $1-4$ and the open-loops with and without close-coupled valve $u_{1}$. Since the $\mathcal{H}_{\infty}$ norm is not defined for unstable systems, it is only depicted for the open-loop stable linearizations. Recall, that the nonzero nominal value of $u_{1}$ enlarges the open-loop stable operating region, as illustrated by Fig. 4 . The optimal NP level with $u_{1}$ is closest to that for the full 10 set, the movable wall $u_{3}$ is second best, and the bleed valve $u_{2}$ is worst. The conclusion that $u_{1}$ is best is in line with the results from [4]. Input $u_{1}$ is the most effective to control the mass flow dynamics (see (5)), which, for the considered case, is considerably faster than the pressure dynamics. The bad results for $u_{2}$ are due to the relatively large plenum volume and small allowable magnitude $\bar{u}_{2}$ : starting from $x_{2}\left(t_{0}\right) \neq 0$, it was observed that the movable wall is much more effective in achieving $x_{2}=0$ than the bleed valve. This may explain why Fig. 4 qualifies $u_{3}$ better than $u_{2}$. Note from this figure, that in the large mass flow region, $\|M\|_{\infty}$ for the closed-loop only improves marginally upon the open-loop. This is due to the open-loop system being stable and with limiting $u$ as dominant performance specification, the computed controller provides only small actions to minimize $z_{1}$ and $z_{2}$.

Figure 5 compares $\|M\|_{\infty}$ for the full IO set 1 , IO sets $5-8$, and the open-loop system with close-coupled valve. The optimal performance with the mass flow sensor $y_{1}$, or the static compressor face pressure sensor $y_{4}$ is the same as with four sensors. The performance with the total compressor face pressure sensor $y_{3}$ is only slightly worse for small mass flows. The plenum pressure rise sensor is worst, since $y_{2}$ is relatively insensitive to the disturbances, due to the large plenum volume. These results fit in with [4], where sensors in the compressor duct (especially $y_{1}$ ) are found the most promising, at least for

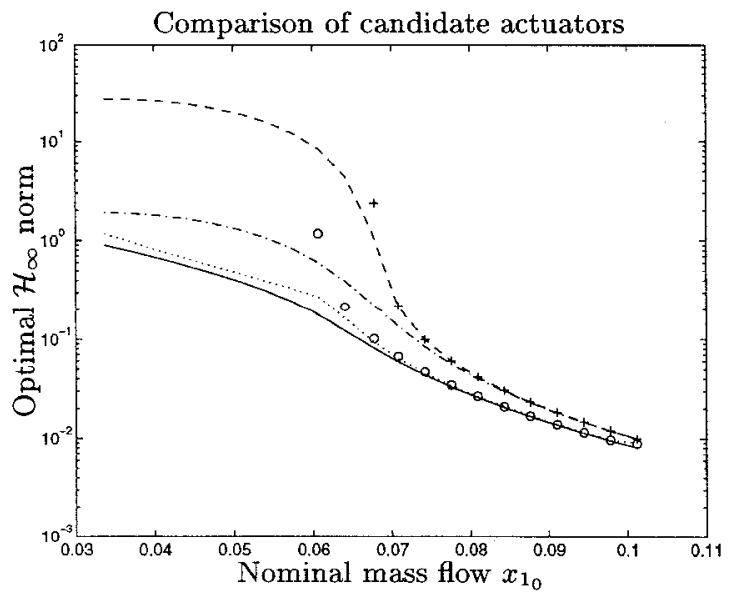

Figure 4: $\|M\|_{\infty}$ from $\mathcal{H}_{\infty}$ optimizations for IO sets 1-4: full IO set $(-)$, IO set $2(\cdot)$, IO set $3(--)$, IO set $4(-\cdot)$, open-loops with $u_{1}(0)$ and without $u_{1}(+)$ 


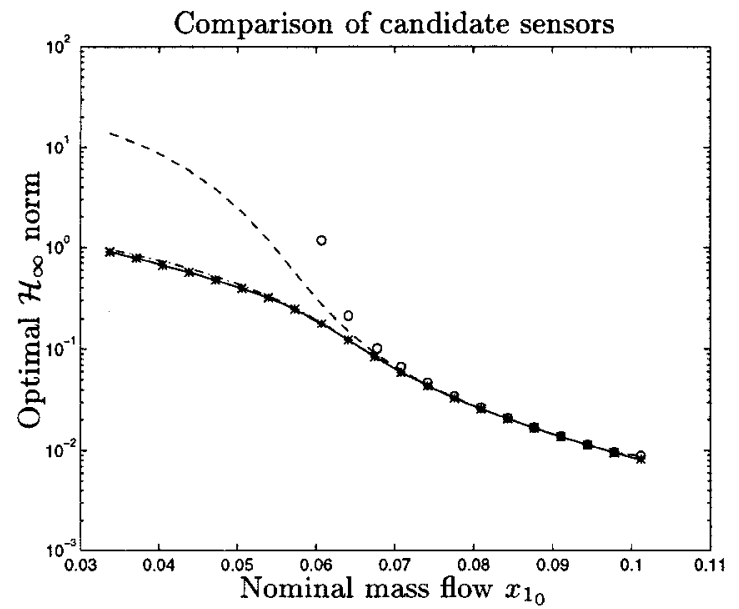

Figure 5: $\|M\|_{\infty}$ from $\mathcal{H}_{\infty}$ optimizations for IO sets 1 and 5-8: full IO set $(-)$, IO set $5(\cdot)$, IO set $6(--)$, IO set $7(-)$, IO set $8(*)$, open-loops with $u_{1}$ (o)

proportional output feedback.

The results in Fig. 4 are strongly affected by actuator limitations, accounted for in $W$ by $\tilde{\omega}_{4}, \tilde{\omega}_{5}$, and $\tilde{\omega}_{6}$ (bandwidth limitations) and $\bar{u}_{1}, \bar{u}_{2}$, and $\bar{u}_{3}$ (allowable magnitudes). $\|M\|_{\infty}$ reduces for increasing bandwidths and allowable magnitudes. In [4] (proportional output feedback), it was already noted that actuator bandwidth considerably affects the possibilities for active surge control. In analogy, the results in Fig. 5 are affected by the sensor noise characterizations $V_{4}, \ldots, V_{7}$. So, with the quantitative performance specifications in $V$ and $W$ it is possible to clearly distinguish between the prospects for candidate IO sets, but inaccurate specifications may give rise to incorrect conclusions. On the other hand, if the specifications are not definite, suitable settings could be found by modifying them so the desired $\mathcal{H}_{\infty}$ norm is achieved. In this way, the designer could, for instance, decide on the actuator bandwidth by manipulating the appropriate parameter in $W$. Whether the specifications are definite or not, it is crucial that they are as well as possible formulated in the $\mathcal{H}_{\infty}$ norm setting.

\section{IO Selection Results}

All 105 candidate IO sets are subjected to the conditions of Section 3. Table 3 summarizes the results. To start the IO selection, the focus is on speed $n_{3}$ and $\gamma=2$, which is more than twice the largest optimal $\mathcal{H}_{\infty}$ norm $\|M\|_{\infty}=0.90$ for the full IO set in the studied operating range. Figure 4 and 5 show that this NP level is even achieved by the uncontrolled system in almost the entire stable mass flow region. Therefore, the IO selection is only performed for the region $x_{1_{0}} \in[F, 2 F]$. All 105 IO sets are first tested for $x_{1_{0}}=F$ and accepted IO sets are tested for larger nominal mass flows. However, for all cases IO selection for $x_{1_{0}}>F$ does not eliminate additional IO sets. This is consistent with Fig. 4 and 5, which show that for the typical 10 sets the largest $\mathcal{H}_{\infty}$ norm occurs at $x_{1_{0}}=F$, where the open-loop's linearization has the largest unstable pole (see also [4], where it is stated that control is more difficult for larger slopes of $\Psi_{c}$ ). Thus, stabilization and disturbance rejection under input constraints is easier for large mass flows. For $n_{3}$ and $\gamma=2,2310$ sets are rejected. Among these are the 15 candidates with the single bleed valve $u_{2}$ and the six candidates based on the single plenum pressure sensor $y_{2}$, which is in line with the results from Fig. 4 and 5. Finally, $y_{3} / u_{1}$ and $y_{3} / u_{1} u_{2}$ are eliminated, where the combination of output set
Table 3: IO selection results

\begin{tabular}{cccc}
\hline Case & $\gamma$ & Speed & Accepted IO sets \\
\hline \hline 1 & 2 & $n_{3}$ & 82 \\
2 & 1 & $n_{3}$ & 28 \\
\hline 3 & 2 & $n_{1}$ & 56 \\
4 & 2 & $n_{2}$ & 80 \\
5 & 2 & $n_{4}$ & 54 \\
6 & 2 & $n_{5}$ & 54 \\
\hline 7 & 2 & $n_{1}, \ldots, n_{5}$ & 28 \\
8 & 3 & $n_{1}, \ldots, n_{5}$ & 49 \\
\hline
\end{tabular}

and input set does not work well (condition 6 fails). For $\gamma=1$ instead of $\gamma=2$, only 28 IO sets are accepted, which all use $u_{1}$ and $u_{3}$ and at least one of $y_{1}, y_{3}$, or $y_{4}$.

IO selection is also performed for lower $\left(n_{1}, n_{2}\right)$ and higher compressor speeds $\left(n_{4}, n_{5}\right)$, see Fig. 2. For the lowest speed $n_{1}, 49$ IO sets are eliminated instead of 23 for $n_{3}$. These are $y_{1} y_{2} y_{3} y_{4} / u_{1} u_{2}$ and its 44 subsets and four additional IO sets based on the single $y_{2}$. This result conflicts with the expectation that control is easier for lower speeds (more general, for lower $B$-parameters [4]). The close-coupled valve $u_{1}$ does not work as well for $n_{1}$ as for $n_{3}$, while the movable wall $u_{3}$ performs better. A possible explanation for this shift in effectiveness is, that the unstable mass flow dynamics (which is directly affected by $u_{1}$, see (5)) becomes slower for lower speeds, while the unstable pressure dynamics (directly affected by $u_{3}$ ) becomes faster. For $n_{2}, 25$ IO sets are eliminated, which are the 15 IO sets using only $u_{2}$, the six additional IO sets based on the single $y_{2}$, and $y_{3} y_{4} / u_{1}, y_{3} / u_{1}, y_{4} / u_{1}$, and $y_{3} / u_{1} u_{2}$. For the higher speeds $n_{4}$ and $n_{5}, 51$ IO sets are rejected which are the same for both speeds: $y_{1} y_{2} y_{3} y_{4} / u_{2} u_{3}$ and its 44 subsets, $y_{2} / u_{1} u_{2} u_{3}$ and its three subsets, $y_{3} / u_{1} u_{2}$, and $y_{3} / u_{1}$.

There are 28 IO sets accepted for $\gamma=2$ and all speeds. These employ both the close-coupled valve $u_{1}$ and the movable wall $u_{3}$ and at least one of the sensors $y_{1}, y_{3}$, or $y_{4}$. Hence, IO sets $y_{1} / u_{1} u_{3}, y_{3} / u_{1} u_{3}$, and $y_{4} / u_{1} u_{3}$ are the smallest accepted candidates. $\mathcal{H}_{\infty}$ optimizations for $n_{1}, \ldots, n_{5}$ in the range $x_{1_{0}} \in[F, 3 F]$ show, that the mass flow sensor $y_{1}$ is the best in combination with $u_{1} u_{3}$. From practical viewpoints, however, either of the pressure sensors $y_{3}$ or $y_{4}$ may be preferred to $y_{1}$. Note, that the IO sets accepted for $\gamma=2$ and all speeds use at least two actuators. To investigate which actuator is most promising for control, IO selection for all speeds is performed with a relaxed NP level $\gamma=3$. The single smallest accepted IO set is then $y_{1} / u_{1}$ with the mass flow sensor and the close-coupled valve.

\section{Closed-Loop Simulations}

For IO sets $y_{1} / u_{1} u_{3}$ and $y_{1} / u_{1}$, simulation results are shown. Results for the full IO set are not depicted, since these are only slightly better than for $y_{1} / u_{1} u_{3}$. So, adding pressure sensors and the bleed valve does not improve the results for the considered case. For speed $n_{3}$ and nominal mass flow $x_{1_{0}}=F$, a linear controller is designed and, starting from stationary operation, the resulting nonlinear closed-loop is subjected to disturbances of the form:

$$
w_{i}= \pm v_{i} \exp \left\{-\tilde{\omega}_{i} \tilde{t}\right\}, \quad i=1,2,3 .
$$

Sensor noise is not incorporated in the simulations. Here, $v_{i}$ and $\tilde{\omega}_{i}$ are parameters in the shaping filters $V_{i}(7)$, which are directly related to the Laplace transforms of the disturbances. So, $w_{i}$ may not be an appropriate representation of a physical 

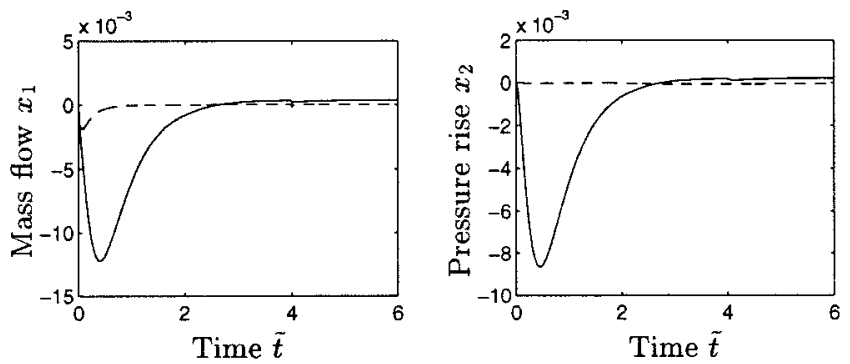

Figure 6: Nonlinear closed-loop responses for $x_{1_{0}}=F$ and speed $n_{3}:(-) y_{1} / u_{1} u_{3},(-) y_{1} / u_{1}$
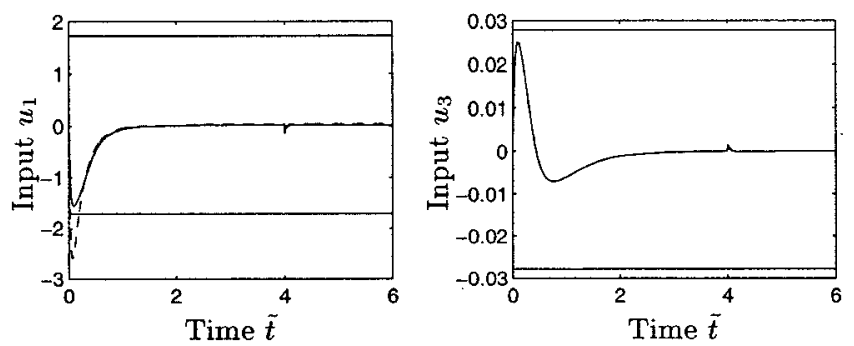

Figure 7: Inputs for nonlinear closed-loop simulations for $x_{1_{0}}=$ $F$ and speed $n_{3}:(-) y_{1} / u_{1} u_{3},(-) y_{1} / u_{1}$

disturbance, but rather is a disturbance belonging to the class modeled by $V_{i}$. In the simulations, $w_{1}, w_{2}$, and $w_{3}$ occur in succession; the nondimensional time between $w_{1}$ and $w_{2}, w_{2}$ and $w_{3}$ is two.

Figures 6 and 7 show the results for disturbances in negative direction, which are worse than for positive direction. The states $x$ and inputs $u$ are the deviations from their nominal values. For $y_{1} / u_{1} u_{3}\left(\|M\|_{\infty}=0.91\right), u$ stays within its limits, but for $y_{1} / u_{1}\left(\|M\|_{\infty}=1.19\right)$ the input bound is exceeded by a factor 1.8. This may be the main reason for the fast disturbance rejection with this IO set. Violation of the input limits cannot be circumvented by increasing $\rho_{1}$ in the weight for $u_{1}$ and setting $W_{1}=W_{2}=0$. Apparently, with $y_{1} / u_{1}$ and for $x_{1_{0}}=F$ it is impossible to jointly stabilize the system and to meet the input constraints in the face of the prescribed set of disturbances. Stabilization and disturbance rejection under the limits on $u_{1}$ can be achieved for nominal mass flows $x_{1_{0}} \geq 1.2 F$.

\section{Discussion}

An IO selection method developed for linear systems was used for a nonlinear compression system. Therefore, the method was applied to linearizations in various nominal operating points. For the investigated problem, the operating point with the smallest nominal mass flow is restrictive, in the sense that other points do not result in elimination of extra IO sets. For the accepted IO sets, subsequent studies (e.g., those suggested in [7]) must lead to definite conclusions on the applicability for the nonlinear system.

To clearly distinguish between the prospects for distinct IO sets, the IO selection method incorporates quantitative performance specifications via the design filters $V$ and $W$. For reliable IO selection results, these specifications should be chosen properly, which must be borne in mind if the disturbances, sensor noises, and actuator limitations are not exactly known or not fixed. This also played a role for the compressor control problem. On the other hand, varying those parameters in $V$ and $W$ which represent actuator and sensor limitations can create insight into the required actuator and sensor quality.

The performance specifications must be formulated in the $\mathcal{H}_{\infty}$ norm setting, which may not be straightforward. For instance, for the compressor control problem the inputs are required to stay within bounds. Ideally, this requirement is directly cast into the weighting filter $W$, but here iterative controller design and simulation had to be invoked to find suitable weights. The development of IO selection methods for mixed-norm control problems merits further research, since many practical problems can be formulated more accurately if there is no restriction to a single system norm.

The close-coupled valve $u_{1}$ and movable wall $u_{3}$ combined with the compressor mass flow sensor $y_{1}$ is the best smallest IO set which achieves the imposed performance specifications. In line with conclusions from [4], $y_{1} / u_{1}$ is the most promising IO set with a single actuator and sensor; $u_{1}$ directly acts upon the mass flow $x_{1}$, while $y_{1}$ directly measures $x_{1}$. The bleed valve $u_{2}$ and the plenum pressure rise sensor $y_{2}$ are the worst candidates among the proposed ones, but they may function better for smaller plenum volumes. For most IO sets, the required NP level is more difficult to achieve for higher compressor speeds, except for IO sets based on the close-coupled valve $u_{1}$. This is probably due to the pressure dynamics (which is not directly affected by $u_{1}$ ) becoming relatively more important for lower speeds. In future research, other sensors and actuators could be investigated, e.g., air injection, which is promising according to [4], or rotor speed manipulation.

For the compression system, various model uncertainties play a role, e.g., uncertain $B$-parameter, uncertain $\Psi_{c}$, and unmodeled dynamics. Also, the linearization errors could be treated as uncertainties. So, a more rigorous control goal is stabilization and guaranteed performance for a class of uncertainties, i.e., robust performance. IO selection methods aiming at this goal are currently under development.

\section{References}

[1] G. J. Balas, J. C. Doyle, K. Glover, A. Packard, and R. Smith. $\mu$-Analysis and synthesis toolbox. The MathWorks, Natick, MA, 1995. Version 3.0.

[2] B. de Jager. Rotating stall and surge control: A survey. In Proc. of the 34th IEEE Conf. on Decision and Control, volume 2, pages 1857-1862, New Orleans, LA, 1995.

[3] E. M. Greitzer. Surge and rotating stall in axial flow compressors. Part I: Theoretical compression system model. ASME J. Engineering for Power, 98(2):190-198, 1976.

[4] J. S. Simon, L. Valavani, A. H. Epstein, and E. M. Greitzer. Evaluation of approaches to active compressor surge stabilization. ASME J. of Turbomachinery, 115:57-67, 1993.

[5] M. van de Wal and B. de Jager. Control structure design: A survey. In Proc. of the 1995 American Control Conf., volume 1, pages 225-229, Seattle, WA, 1995.

[6] M. van de Wal and B. de Jager. Selection of sensors and actuators for an active suspension control problem. In Proc. of the 1996 IEEE Internat. Conf. on Control Applications, pages 55-60, Dearborn, MI, 1996.

[7] M. van de Wal and F. Willems. Selection of actuators and sensors for compressor control. Technical Report WFW 96.155, Fac. of Mechanical Engineering, Eindhoven University of Technology, 1996. 67 pages, request by email.

[8] A. J. van der Schaft. $\mathcal{L}_{2}$-gain and passivity techniques in nonlinear control, volume 218 of Lecture Notes in Control and Information Sciences. Springer-Verlag, Heidelberg, 1996.

[9] K. Zhou, J. C. Doyle, and K. Glover. Robust and optimal control. Prentice-Hall, Upper Saddle River, NJ, 1996. 